ARTICLE OPEN

\title{
Characterizing decoherence rates of a superconducting qubit by direct microwave scattering
}

Yong Lu (iD ${ }^{1 凶}$, Andreas Bengtsson (iD) ${ }^{1}$, Jonathan J. Burnett ${ }^{1,2}$, Emely Wiegand ${ }^{1}{ }^{1}$, Baladitya Suri ${ }^{1,3}$, Philip Krantz ${ }^{1}$, Anita Fadavi Roudsari (iD), Anton Frisk Kockum ${ }^{1}$, Simone Gasparinetti $\mathbb{D}^{1}$, Göran Johansson ${ }^{1}$ and Per Delsing (iD ${ }^{1 凶}$

We experimentally investigate a superconducting qubit coupled to the end of an open transmission line, in a regime where the qubit decay rates to the transmission line and to its own environment are comparable. We perform measurements of coherent and incoherent scattering, on- and off-resonant fluorescence, and time-resolved dynamics to determine the decay and decoherence rates of the qubit. In particular, these measurements let us discriminate between non-radiative decay and pure dephasing. We combine and contrast results across all methods and find consistent values for the extracted rates. The results show that the pure dephasing rate is one order of magnitude smaller than the non-radiative decay rate for our qubit. Our results indicate a pathway to benchmark decoherence rates of superconducting qubits in a resonator-free setting.

npj Quantum Information (2021)7:35; https://doi.org/10.1038/s41534-021-00367-5

\section{INTRODUCTION}

Superconducting circuits are promising building blocks for implementing quantum computers ${ }^{1-3}$. In those devices, the key elements are superconducting artificial atoms made by Josephson junctions which induce a strong and engineerable nonlinearity. Such artificial atoms are also used in the field of superconducting waveguide quantum electrodynamics (waveguide QED) ${ }^{4,5}$, where they interact with a continuum of light modes in a 1D waveguide. In the past decade, many quantum effects from atomic physics and quantum optics have been demonstrated in waveguide QED, e.g., the Mollow triplet ${ }^{6}$, giant cross-Kerr effect ${ }^{7}$, and cooperative effects $^{5,8,9}$. Other recent experiments have shown phenomena which are currently beyond the reach of atomic physics, such as ultra-strong $^{10,11}$ and superstrong coupling ${ }^{12}$ between light and matter. Waveguide QED is also an enabling quantum technology. One of the key applications is to generate ${ }^{13-19}$ and detect ${ }^{4,20-26}$ single photons. It has been proposed to use waveguide QED to create bound states ${ }^{27-29}$ and implement quantum computers ${ }^{30-32}$.

The performance of quantum computers and waveguide-QED devices is often limited by the coherence of the Josephson circuits. For example, the efficiency of producing and detecting single photons, the lifetime of bound states, and the fidelity of logical gates can all be improved by enhancing the coherence. In a waveguide-QED setup, decoherence can be due to decay into the waveguide, pure dephasing, and non-radiative decay into other modes. However, the rates for pure dephasing and nonradiative decay are typically not explored separately. An understanding of which one is dominant will give an insight into the decoherence mechanisms, and thus how the device performance can be improved.

In this work, we probe a superconducting transmon qubit coupled directly to the end of an open transmission line. In previous realizations ${ }^{6-8,33-35}$, the coupling rates were much larger than intrinsic decoherence mechanisms of the qubit, so the effects of non-radiative decay and pure dephasing were small and could not be well characterized. Here, we investigate a qubit whose radiative decay rate into the transmission line is larger than, yet comparable to, other decoherence mechanisms. This allows us to explore the pure dephasing rate $\Gamma_{\phi}$, the radiative decay rate $\Gamma_{r}$ from the capacitive coupling to the waveguide, and the nonradiative decay rate $\Gamma_{\mathrm{n}}$. The total relaxation and decoherence rates are given by $\Gamma_{1}=\Gamma_{\mathrm{r}}+\Gamma_{\mathrm{n}}$ and $\Gamma_{2}=\Gamma_{1} / 2+\Gamma_{\phi}$, respectively. We demonstrate different methods to extract the different rates and find consistent results. Similar to the results in circuit QED ${ }^{36-40}$, our methods can also enable the evaluation of the decoherence of qubits over a broad range of frequencies. They provide a pathway to investigate Josephson junctions or superconducting quantum interference devices (SQUIDs) without any resonator. In addition, we also consider it important to study $\Gamma_{\mathrm{n}}$ and $\Gamma_{\phi}$ separately. For instance, this could help to improve the Purcell enhancement factor, $\frac{\Gamma_{\mathrm{r}}}{\Gamma_{\mathrm{n}}+2 \Gamma_{\phi}}$ in devices such as the one presented in ref. ${ }^{9}$. Moreover, the spontaneous-emission factor $\beta$, which is customarily quoted in other waveguide-QED platforms ${ }^{41-44}$ is also related to $\Gamma_{\mathrm{n}}$, namely, $\beta=\frac{\Gamma_{\mathrm{r}}}{\Gamma_{\mathrm{r}}+\Gamma_{\mathrm{n}}}$ in our case.

The paper is structured as follows: first, we characterize the coherent scattering of the device and obtain the radiative decay rate and the decoherence rate of the qubit as a reference for later measurements. Then, we exploit the fluorescence of the qubit under coherent excitation to find the non-radiative decay rate and the pure dephasing rate. The resonance fluorescence spectrum at strong driving develops into the Mollow triplet ${ }^{45}$, which has been widely used to probe quantum properties in systems based on superconducting qubits such as coherence ${ }^{6,8}$ and vacuum squeezing ${ }^{46}$. The resonance spectrum is symmetric around the central peak. However, if pure dephasing exists, the off-resonant spectrum becomes asymmetric, something which has been studied experimentally in quantum dots ${ }^{47,48}$. We take advantage of this fact to extract the pure dephasing rate. We also measure the non-radiative decay rate under a continuous coherent drive, where coherently and incoherently scattered photons provide information about the different decay channels. Afterward, we apply a pulse to the qubit to both obtain the decay rates and find the stability of the qubit frequency and coherence as a function of time. In contrast to other methods, we use the phase information

${ }^{1}$ Department of Microtechnology and Nanoscience (MC2), Chalmers University of Technology, Göteborg, Sweden. ${ }^{2}$ National Physical Laboratory, Hampton Road, Teddington, Middlesex, UK. ${ }^{3}$ Indian Institute of Science, Department of Instrumentation and Applied Physics, Bangalore, India. ${ }^{凶}$ email: yongl@chalmers.se; per.delsing@chalmers.se 
of emitted photons from the qubit to investigate the qubitfrequency stability in superconducting waveguide QED. Finally, we summarize the measured results and compare the advantages and disadvantages of the different methods.

In this paper, we have investigated waveguide QED with a qubit weakly coupled to a transmission line which is an unexplored regime. In this regime, it becomes possible to quantify the nonradiative decay rate of the qubit. We use several different methods to extract the different decay rates, provide a thorough comparison of the different methods, and find consistent results. Among these methods, those based on the off-resonant Mollow triplet and the power loss analysis are original. Moreover, we show that time-domain analysis of decay rates can also be done in waveguide QED.

\section{RESULTS}

\section{Device characterization}

The device used in our experiment (see Fig. 1a) is a magnetic-fluxtunable Xmon-type transmon qubit ${ }^{49}$, capacitively coupled to the open end of a one-dimensional transmission line with characteristic impedance $Z_{0} \simeq 50 \Omega$. The circuit is equivalent to an atom in front of a mirror in 1D space. The device is fabricated from aluminum on a silicon substrate using the same fabrication recipe as in ref. ${ }^{38}$. We denote $|0\rangle,|1\rangle$ and $|2\rangle$ as the ground state, the first and the second excited states of the qubit, respectively. The $|0\rangle-$ $|1\rangle$ transition energy is $\hbar \omega_{01} \approx \sqrt{8 E_{J}(\Phi) E_{C}}-E_{C}$, where $E_{\mathrm{C}}=e^{2}$ / $\left(2 C_{\Sigma}\right)$ is the charging energy, $e$ is the elementary charge, $C_{\Sigma}$ is the total capacitance of the qubit, and $E_{J}(\Phi)$ is the Josephson energy. The Josephson energy can be tuned from its maximum value $E_{J, \text { max }}$ by an external magnetic flux $\Phi$ using a coil: $E_{J}(\Phi)=E_{J, \max }\left|\cos \left(\pi \Phi / \Phi_{0}\right)\right|$, where $\Phi_{0}=h /(2 e)$ is the magnetic flux quantum.

Figure 1a illustrates the simplified experimental setup for measuring the reflection coefficient of a probe signal coming from a vector network analyzer (VNA), after interacting with the qubit. The probe signal at frequency $\omega_{\mathrm{pr}}$ and a pump at frequency $\omega_{p}$ are combined and attenuated before being fed into the transmission line. Then, the VNA receives the reflected signal to determine the complex reflection coefficient.

Figure $1 \mathrm{~b}$ shows the magnitude of the reflection coefficient, $|r|$, for a weak probe (with an intensity $\Omega_{\mathrm{pr}}<\Gamma_{2}$ ) as a function of the external flux $\Phi$. Afterward, all the other measurements are taken at the flux-sweet spot. We use two-tone spectroscopy to determine the anharmonicity of the qubit, $a=\left(\omega_{12}-\omega_{01}\right) / 2 \pi$, where $\omega_{12}$ is the frequency of the $|1\rangle-|2\rangle$ transition. Specifically, we apply a strong pump (with an intensity $\Omega_{p} \gg \Gamma_{2}$ ) at $\omega_{01}$ to saturate the $|0\rangle-|1\rangle$ transition and measure the reflection coefficient as a function of probe frequency. The result is shown in Fig. 1c: a dip appears in the reflection at $\omega_{\mathrm{pr}}=\omega_{12}$ due to the photon scattering from the $|1\rangle-|2\rangle$ transition. At a higher pump power, the Autler - Townes splitting is observed ${ }^{50}$. From Fig. $1 \mathrm{~b}, \mathrm{c}$, we find $E_{\mathrm{C}} / h \approx a$ $=252 \mathrm{MHz}$, and then, $E_{\mathrm{J}, \max } / h=16.56 \mathrm{GHz}$ by fitting the data in Fig. $1 b$ to the equation between the qubit frequency and the external flux mentioned previously. In order to obtain the radiative decay and decoherence rates, we perform single-tone spectroscopy with a weak probe $\left(\Omega_{\mathrm{pr}} \ll \Gamma_{2}\right)$. Figure $1 \mathrm{~d}$, e are the corresponding magnitude and phase response of $r$, where we obtain $\Gamma_{\mathrm{r}} / 2 \pi=227 \mathrm{kHz}$ and $\Gamma_{2} / 2 \pi=141 \mathrm{kHz}$ by using the circle fit technique from ref. ${ }^{51}$. The corresponding fitting equation is

$r=1-\frac{\mathrm{i}_{\mathrm{r}} \mathrm{e}^{\mathrm{i} \phi}}{\Delta+\mathrm{i} \Gamma_{2}}$

where the phase $\phi \approx 0.150 \pm 0.004$ quantifies the impedance mismatches in the line (for a perfectly matched line, $\phi=0$ ).
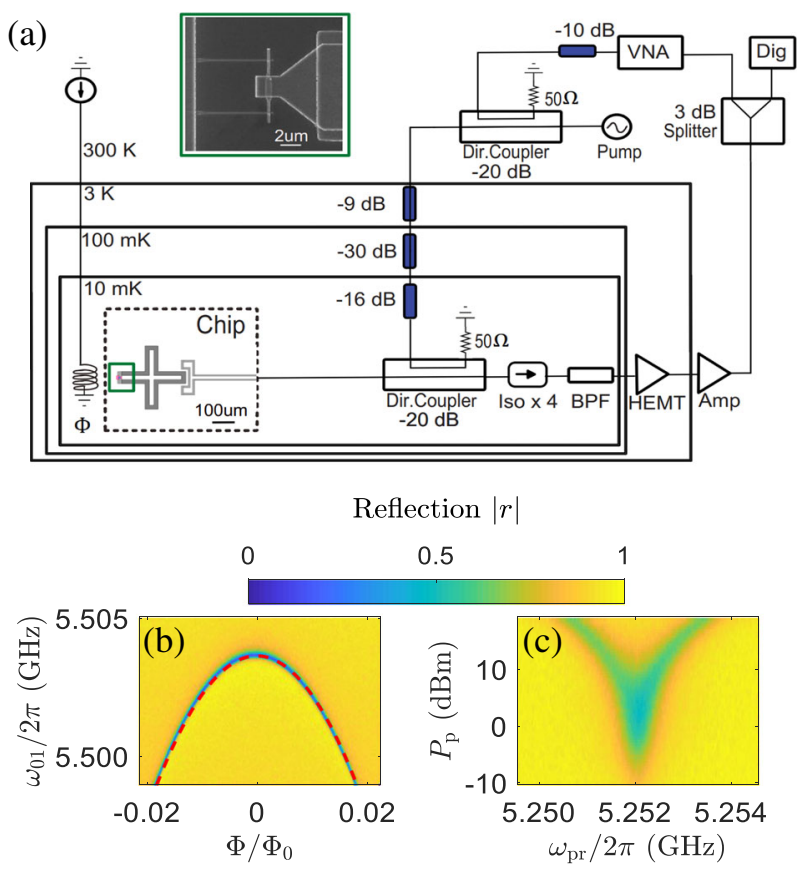

Reflection $|r|$

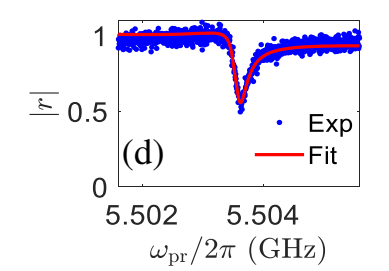

0.5

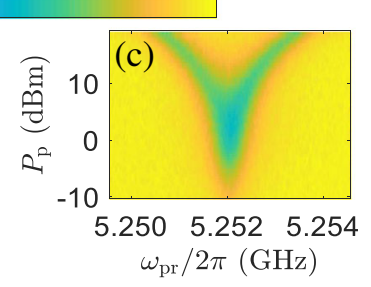

Fig. 1 Measurement setup and spectroscopy of a transmon qubit. a A simplified schematic of the setup and experimental device. Iso, BPF, HEMT, Amp, and Dig denote isolators, a bandpass filter, a high electron mobility transistor amplifier, room-temperature amplifiers, and a digitizer, respectively. Our chip CAD design is shown inside the dashed box, where a qubit is formed by a cross-shaped island connected to the ground plane via two Josephson junctions located in the center of the green box. The scale bar in the bottom-right side is $100 \mu \mathrm{m}$. Top inset: scanning electron micrograph of the Josephson junctions. The corresponding scale bar in the bottom right is $2 \mu \mathrm{m}$. b Single-tone spectroscopy. The magnitude of the reflection coefficient $r$ is measured as a function of the external flux $\Phi$ and probe frequency $\omega_{\mathrm{pr}}$. The red dashed curve is a fit for the qubit frequency $\omega_{01}$. c Two-tone spectroscopy. A strong pump is applied to the $|0\rangle-|1\rangle$ transition and we sweep the frequency of the weak probe on the $|1\rangle-|2\rangle$ transition to obtain the corresponding reflection coefficient. d, e show the magnitude and phase response of the qubit at $\Phi=0$ under weak probing. Red lines are the corresponding fits using the circle fit technique from ref. ${ }^{51}$.

\section{Atomic fluorescence}

Even though measuring the reflection coefficient can give the decoherence and radiative decay rates, it cannot distinguish between pure dephasing and non-radiative decay. In order to distinguish them, we study the atomic fluorescence for different pump intensities and frequencies. For this measurement, the VNA is turned off, the pump is used to drive the system, another $50 \mathrm{~dB}$ of attenuation is added between the directional coupler and the pump, and the output signal is sampled by a digitizer (see Fig. 1a). When the qubit is pumped, its state evolves at a Rabi frequency $\Omega$. With a Rabi frequency much larger than the natural linewidth of the qubit $\left(\Omega \gg \Gamma_{2}\right)$, the energy levels of the qubit become dressed, leading to three distinct spectral components known as the Mollow triplet ${ }^{45}$. In particular, the spectrum contains the elastic "Rayleigh" line in the middle in which the scattered wave has the 
(a)

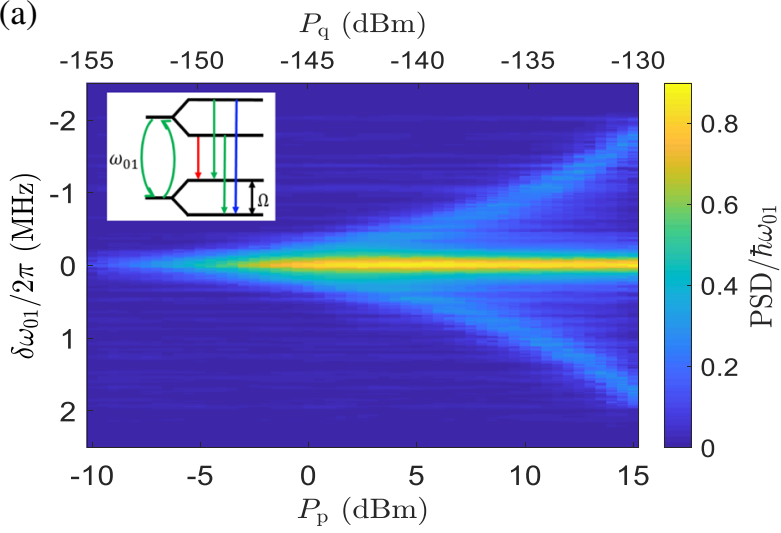

(c)

(b)
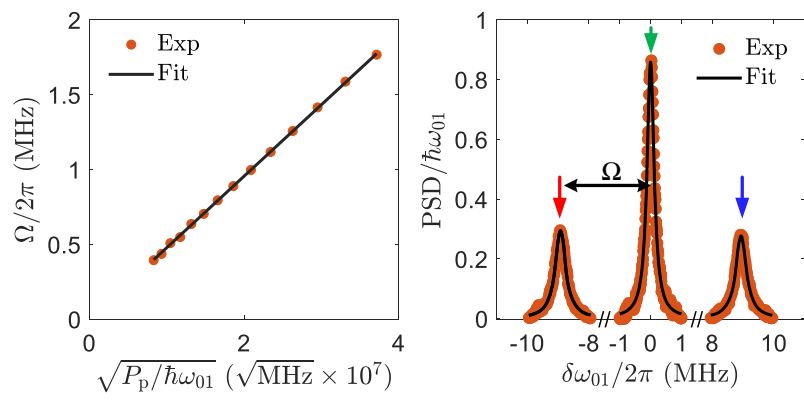

Fig. 2 Power spectral density (PSD) of the on-resonant Mollow triplet from the atomic fluorescence. a Resonant fluorescence emission spectrum as a function of the pump power and detuning of the detected radiation, $\delta \omega_{01}=\omega-\omega_{01} . P_{\mathrm{p}}$ is the power from the RF source while $P_{\mathrm{q}}$ is the corresponding power on the qubit. Inset: a schematic of the triplet transitions in the dressed-state picture, where the qubit energy levels split by $\Omega$ due to strong driving, creating three transitions with frequencies $\omega_{01}-\Omega, \omega_{01}$ and $\omega_{01}+\Omega$. b Rabi splitting $\Omega$ (dots) vs. drive amplitude, extracted from (a). The black line is the linear fit to obtain the attenuation in the input line which is $A=-145 \mathrm{~dB}$. c Power spectral density at $-116 \mathrm{dBm}$ power at the sample. The black lines are individual fits to the linewidths of the three peaks, yielding $\Gamma_{2} / 2 \pi=141 \pm 2 \mathrm{kHz}$ and $\Gamma_{1} / 2 \pi=276 \pm$ $5 \mathrm{kHz}$. The arrows correspond to the transitions in the inset of (a).

same frequency as the incident wave, with two inelastic sidebands positioned symmetrically on both sides of the central peak.

To measure the power spectrum for the atomic fluorescence, we use a digitizer to sample the amplified signal as $V(t)$ in the time domain. The sample rate and the number of samples are from $2 \mathrm{MHz}$ to $6 \mathrm{MHz}$ and from 500 to 1000 , respectively, for the different measured spectra in Figs. 2 and 3. After that, we calculate the power spectrum $S_{i}^{\text {meas. }}(\omega)$ according to the Welch method ${ }^{52}$, after normalization to the system gain $G$. In order to increase the corresponding signal-to-noise ratio (SNR), a number of averages are required to obtain $\left\langle S_{i}^{\text {meas. }}(\omega)\right\rangle$. For different results shown below, the number of averages varies from $2 \times 10^{6}$ to $10^{8}$.

\section{On-resonant Mollow triplet}

When the qubit is driven by a resonant pump $\left(\Delta=\omega_{\mathrm{p}}-\omega_{01}=0\right)$, as shown in Fig. 2a, the splitting of the spectrum between the sidebands and the central peak increases as a function of the pump power $P_{\mathrm{p}}$. The splitting equals the Rabi frequency and obeys $\Omega=2 \sqrt{A \Gamma_{\mathrm{r}} P_{\mathrm{p}} /\left(\hbar \omega_{01}\right)}$. By fitting the extracted Rabi splitting $|\Omega|$ in Fig. $2 b$, and using $\Gamma_{\mathrm{r}}$ from the previous measurement in the section "Device characterization", we extract a total attenuation $A=-145 \mathrm{~dB}$ of which about $-125 \mathrm{~dB}$ attenuation is from attenuators and directional couplers, $-7 \mathrm{~dB}$ from an Eccosorb (a)

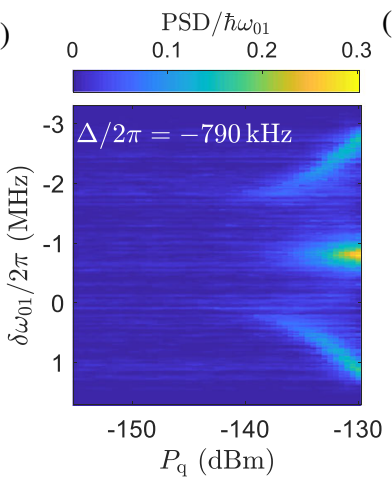

(b)
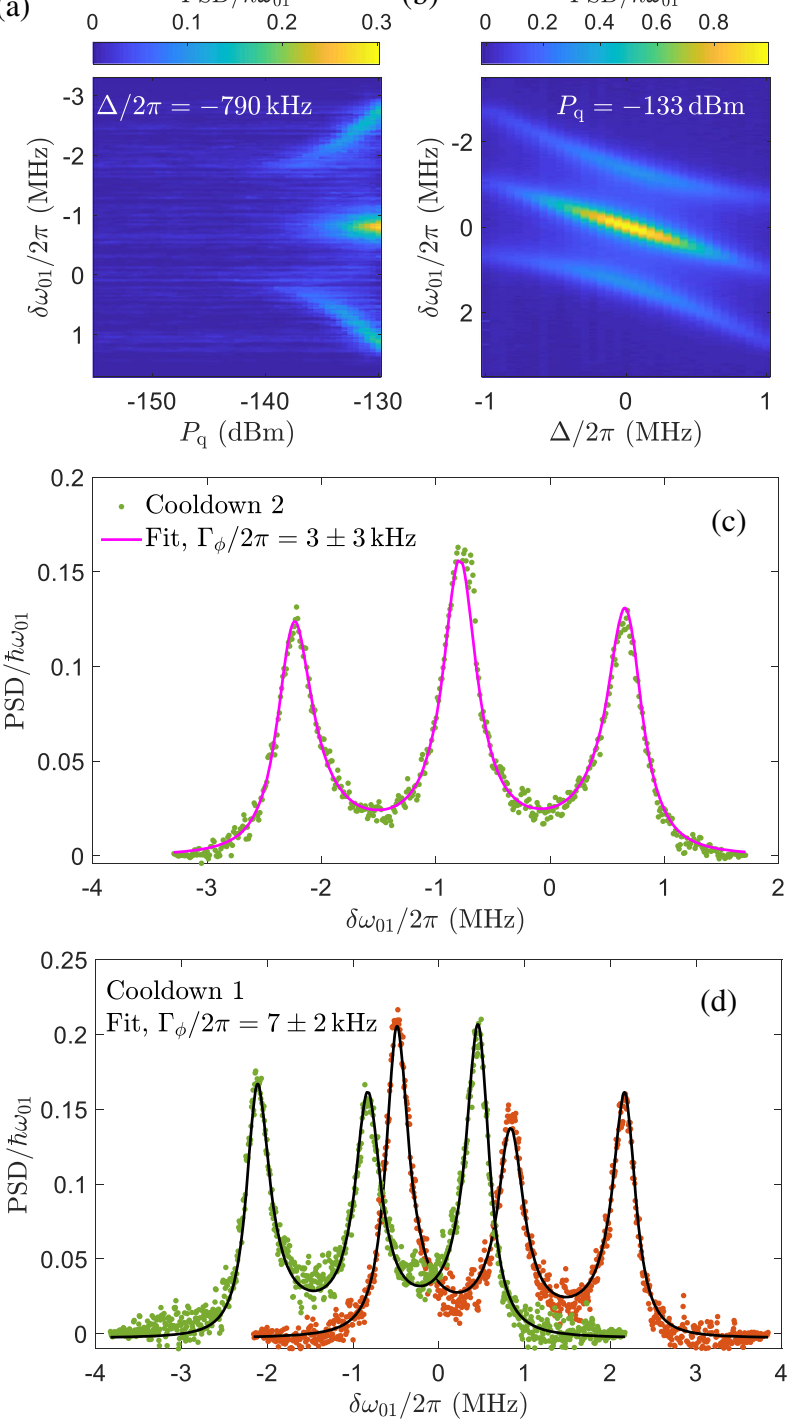

Fig. 3 Power spectral density of the off-resonant Mollow triplet from the atomic fluorescence. a Off-resonant fluorescence emission spectrum as a function of the pump power at the qubit. The frequency of the pump field is detuned by $\Delta=\omega_{\mathrm{p}}-\omega_{01}=$ $-2 \pi^{*} 790 \mathrm{kHz}$ from the qubit frequency. The Rabi splitting is increased with the input power. b Off-resonant fluorescence emission spectrum as a function of the frequency of the pump at $P_{\mathrm{q}}=-133 \mathrm{dBm}$. c Off-resonant PSD at $\Delta / 2 \pi=-790 \mathrm{kHz}$ (green dots) with $P_{\mathrm{q}}=-133 \mathrm{dBm}$. The solid curve is a fit to $\Gamma_{1} / 2 \pi=275 \pm 6 \mathrm{kHz}$ and $\Gamma_{\phi} / 2 \pi=3 \pm 3 \mathrm{kHz}$. d Off-resonant PSD in a second cooldown at $\Delta / 2 \pi=820 \mathrm{kHz}$ (brown dots), $\Delta / 2 \pi=-830 \mathrm{kHz}$ (green dots). A value of $\Gamma_{\phi} / 2 \pi=7 \mathrm{kHz}$ gives a good fit to both traces (black).

filter and the rest is due to cable loss. This allows us to renormalize all applied powers to either the power at the qubit, or the corresponding Rabi frequency. The total gain in the output line of the measurement setup can be calibrated by tuning the qubit away and measuring the power at the output port at room temperature. This results in a total gain $G=115 \mathrm{~dB}$, of which approximately $44 \mathrm{~dB}$ gain comes from a high electron mobility transistor (HEMT) amplifier, and the rest is from the room temperature amplifiers and the pre-amplifiers of the digitizer.

The Rabi rate can be made much larger than all the decay rates of the qubit $\left(\Omega \gg \Gamma_{1}, \Gamma_{2}\right)$. Consequently, the overlap in the frequency domain between the sideband emission and the central peak becomes negligible. In Fig. 2c, we use an input 
power to the qubit $P_{\mathrm{q}} \approx-116 \mathrm{dBm}$, equivalent to $\Omega / 2 \pi \approx 9 \mathrm{MHz}$. The incoherent part of the corresponding power spectral density (PSD) is given by

$$
S_{\mathrm{i}}(\omega) \approx \frac{1}{2 \pi} \frac{\hbar \omega_{01} \Gamma_{\mathrm{r}}}{4}\left\{\frac{\Gamma_{\mathrm{s}}}{\left(\delta \omega_{01}+\Omega\right)^{2}+\Gamma_{\mathrm{s}}^{2}}+\frac{2 \Gamma_{2}}{\delta \omega_{01}^{2}+\Gamma_{2}^{2}}+\frac{\Gamma_{\mathrm{s}}}{\left(\delta \omega_{01}-\Omega\right)^{2}+\Gamma_{s}^{2}}\right\},
$$

(see more details in Supplementary section A), where the half width at half maximum of the central peak and the sidebands are $\Gamma_{2}$ and $\Gamma_{\mathrm{s}}=\left(\Gamma_{1}+\Gamma_{2}\right) / 2$, respectively. The solid curves in Fig. $2 \mathrm{c}$ are fits to $2 \pi S_{i}(\omega)$ which is the PSD expressed in linear frequency. We obtain $\Gamma_{2} / 2 \pi=141 \pm 2 \mathrm{kHz}$ for the central peak, $\Gamma_{\mathrm{s}, \text { red }} / 2 \pi=210 \pm$ $3 \mathrm{kHz}$ and $\Gamma_{\mathrm{s}, \text { blue }} / 2 \pi=206 \pm 4 \mathrm{kHz}$ for sidebands. By taking the average of $\Gamma_{\mathrm{s}, \text { red }}$ and $\Gamma_{\mathrm{s}, \mathrm{blue}}$, we obtain $\Gamma_{1} / 2 \pi=275 \pm 7 \mathrm{kHz}$. We note that the extracted $\Gamma_{2} / 2 \pi$ value is fully consistent with the result from the reflection-coefficient measurement in the section "Device characterization". From that measurement, we also know $\Gamma_{\mathrm{r}} / 2 \pi=227 \pm 1 \mathrm{kHz}$. Thus, we can now extract both the nonradiative decay rate, $\Gamma_{\mathrm{n}} / 2 \pi=48 \pm 7 \mathrm{kHz}$, and the pure dephasing rate, $\Gamma_{\phi} / 2 \pi=3 \pm 4 \mathrm{kHz}$.

By integrating the PSD of each peak in the Mollow triplet we can compare their relative weights. After normalization with $\hbar \omega_{01} \Gamma_{r}$, the results are about $0.254,0.116$ and 0.124 for the middle peak, the red and blue sidebands, respectively. According to Eq. (2), we would expect these numbers to be $0.250,0.125$ and 0.125 , respectively, for a fully saturated qubit.

\section{Off-resonant Mollow triplet}

We also study the off-resonant Mollow triplet at a variety of pump powers and frequency detunings between the pump and the qubit. In Fig. 3a, the pump power at the qubit is swept from $-150 \mathrm{dBm}$ to $-130 \mathrm{dBm}$ at detuning $\Delta / 2 \pi=790 \mathrm{kHz}$. We find that the PSD is weaker than the on-resonant case, implying that the qubit is less excited. In Fig. $3 b$, as we sweep the frequency detuning between the pump and the qubit, the spectrum is nearly symmetric over $1 \mathrm{MHz}$ bandwidth, therefore, the extracted pure dephasing rates by the off-resonant fluorescence are insensitive to the frequency detuning $\Delta$. We can either choose a large $\Delta$ which will lead to a small excitation of the qubit, or a small $\Delta$ which results in an unresolved spectrum between the central peak and sidebands.

Compared to the on-resonant case, the off-resonant Mollow triplet carries additional information in its sideband asymmetry and the approximation used in Eq. (2) is no longer valid. Therefore, the full expression for the PSD must be used, shown in Eq. (16) in Supplementary section A which is an extension of ref. ${ }^{53}$. The fit of the data in Fig. $3 c$ to Eq. (16) yields $\Gamma_{\phi} / 2 \pi=3 \pm 3 \mathrm{kHz}$ (pink solid line). The symmetry of the sidebands around the central peak is due to the relatively small pure dephasing rate. In Fig. 3d, the sample was measured in an earlier cooldown. There, we observed a larger asymmetry for both positive (green dots) and negative detunings (brown dots). In the case of positive detuning, the red sideband is closer to the qubit original frequency than the blue sideband, whereas the blue sideband is closer when the sign of the detuning is changed. We fit the two sets of data simultaneously to obtain $\Gamma_{\phi} / 2 \pi=(7 \pm 2) \mathrm{kHz}$, which is slightly larger than the second cooldown. This is likely due to that we used only two isolators in the first cooldown, and four isolators in the second cooldown, leading to less thermal photons from the transmission line in the second case.

The mechanism by which the pure dephasing gives rise to an asymmetry in the Mollow triplet can be explained as follows (for details, see Supplementary section B). Relaxation from the qubit will cause transitions between dressed states $|n, \pm\rangle$ (see Fig. 4a) that contain different numbers $n$ of drive photons. As shown in Fig. $4 \mathrm{~b}$, these transitions will either be between or within the + and - subspaces. In equilibrium, if the pure dephasing rate is zero, the probabilities $P_{ \pm}$for the system to be in these subspaces are

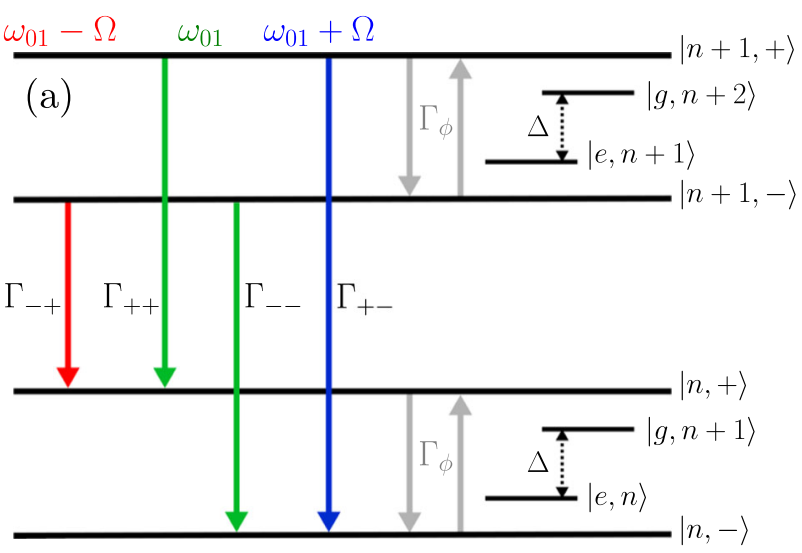

(b)

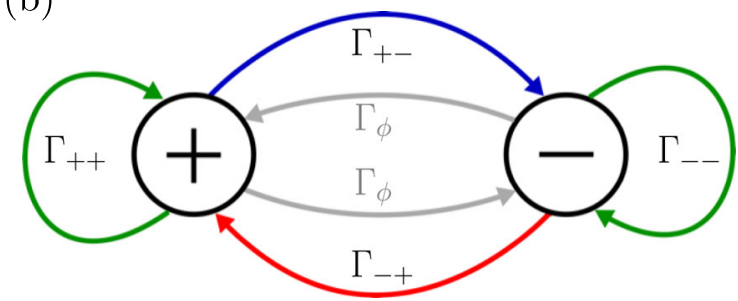

Fig. 4 Dressed states and transitions of the driven qubit. a Sketch of the dressed-state picture, including energies and transition rates. The states $|e, n\rangle,|g, n+1\rangle,|e, n+1\rangle$, and $|g, n+2\rangle$ are the bare states; the states $|n, \pm\rangle$ and $|n+1, \pm\rangle$ are the dressed states. b Transitions and transition rates between the + and - subspaces.

given by the detailed-balance condition

$\Gamma_{+-} P_{+}=\Gamma_{-+} P_{-}$,

i.e., the number of emitted photons causing transitions from + to - (the blue sideband) must equal the number of emitted photons causing transitions from - to + (the red sideband). However, the interaction causing pure dephasing has a non-zero matrix element for transitions between $|n,+\rangle$ and $|n,-\rangle$, which leads to a modified detailed-balance condition:

$\left(\Gamma_{+-}+\Gamma_{\phi}\right) P_{+}=\left(\Gamma_{-+}+\Gamma_{\phi}\right) P_{-}$.

As $\Gamma_{\phi}$ increases, this will push the occupation probabilities towards $P_{+}=P_{-}$. For off-resonant driving $\Gamma_{+-} \neq \Gamma_{-+}$and thus the number of emitted photons in the two sidebands becomes different: $\Gamma_{+}$ ${ }_{-} P_{+} \neq \Gamma_{-+} P_{-}$. The larger number of photons will be emitted at the frequency corresponding to the larger of the two transition rates $\Gamma_{+-}$and $\Gamma_{-+} ;$from transition-matrix elements, this can be seen to be the frequency that is closer to the qubit frequency.

From Fig. $3 c$, we have $\Gamma_{1} / 2 \pi=275 \pm 6 \mathrm{kHz}$ and $\Gamma_{2} / 2 \pi=140 \pm$ $3 \mathrm{kHz}$. Again, from the measured radiative decay rate, by subtracting $\Gamma_{\mathrm{r}}$ from $\Gamma_{1}$, we obtain $\Gamma_{\mathrm{n}} / 2 \pi=48 \pm 6 \mathrm{kHz}$. Based on the results in this section, the on/off-resonant Mollow spectra allow us to extract the pure dephasing rate and non-radiative rate of a qubit. Specifically, for our qubit in this environment, we find that the non-radiative decay rate is one order of magnitude larger than the pure dephasing rate. Compared to the on-resonant Mollow triplet, the off-resonant Mollow triplet allows us to characterize the qubit decay rates at a lower pump power.

\section{Photon scattering by the qubit}

To verify the extracted decay rates above, we can also measure the power scattered by the qubit and the dissipated power due to the non-radiative decay channel directly. We normalize all the powers by the single-photon energy $\hbar \omega_{01}$. The pump is on resonance with the qubit. The output power then consists of a 
coherent part and an incoherent part, $P_{\text {out }}=P_{\text {coh }}+P_{\text {incoh, }}$ where $P_{\text {coh }}=\frac{\Omega^{2}}{4 \Gamma_{\mathrm{r}}}\left(1-\frac{\Gamma_{1} \Gamma_{\mathrm{r}}}{\Omega^{2}+\Gamma_{2} \Gamma_{1}}\right)^{2}$ and $P_{\text {incoh }}=\frac{\Gamma_{\mathrm{r}}}{2} \frac{\Omega^{2}\left(\Gamma_{1} \Gamma_{\phi}+\Omega^{2}\right)}{\left(\Gamma_{1} \Gamma_{2}+\Omega^{2}\right)^{2}}$ (see Supplementary section D).

For our qubit, the pure dephasing rate was verified to be around $3 \mathrm{kHz}$, i.e., much less than other rates and therefore negligible, so, the expression for the incoherent power can be further simplified to $P_{\text {incoh }} \simeq 2 \Gamma_{\mathrm{r}} \rho_{11}^{2}$, where $\rho_{11}$ is the population of the first-excited state of the qubit. In this case, the expression for the dissipated power due to the non-radiative decay is then $P_{\text {loss }}=\Gamma_{\mathrm{n}} \rho_{11}=\Gamma_{\mathrm{n}} \frac{\Omega^{2}}{2\left(\Gamma_{1} \Gamma_{2}+\Omega^{2}\right)}$.

Experimentally, we use about $4.2 \times 10^{9}$ averages to measure all the powers. We denote the measured voltage $V$ and the pump power $P_{\text {in }}$. The subscripts "off" and "on" used in the following contexts mean that the qubit is off/on resonance with the pump, respectively. When the qubit is tuned away, it is off-resonant with the pump; we will have $P_{\text {in }}=\langle V\rangle_{\text {off }}^{2}$ because of the coherence of the pump. Besides the pump power, the system noise will also make a contribution to the total measured power, $P_{\text {in }}^{\text {meas. }}$. Therefore, we have $P_{\text {in }}^{\text {meas. }}=\left\langle V^{2}\right\rangle_{\text {off }}=P_{\text {in }}+P_{\text {noise, }}$, where $P_{\text {noise }}$ is the noise power added by the amplification chain over the measurement bandwith. When instead the qubit is on resonance with the pump, the total measured output power $P_{\text {out }}^{\text {meas. }}=\left\langle V^{2}\right\rangle_{\text {on }}=P_{\text {out }}+P_{\text {noise, }}$ where $P_{\text {coh }}=\langle V\rangle_{\text {on }}^{2}$ (Fig. 5, black circles). Therefore, $P_{\text {loss }}$ (blue crosses) is obtained by taking $P_{\text {loss }}=$ $P_{\text {in }}^{\text {meas. }}-P_{\text {out }}^{\text {meas. }}=P_{\text {in }}-P_{\text {out }}$ with $P_{\text {incoh }}=P_{\text {in }}-P_{\text {coh }}-P_{\text {loss }}$ (green circles). Figure 5 shows all the types of measured power as a function of the Rabi frequency. There, we find three interesting regions:

(i) At high input power, when $\Omega>\left(1+\frac{1}{\sqrt{2}}\right) \Gamma_{\mathrm{r}} \approx 2 \pi * 391 \mathrm{kHz}$, to the right of the dashed line in Fig. 5, the qubit starts to be saturated. The outgoing field is then mainly coherent from the pump itself. By increasing the input power further, the qubit is completely saturated, leading to $P_{\text {in }} \approx P_{\text {coh }} \approx \frac{\Omega^{2}}{4 \Gamma_{r}{ }^{\prime}}$ $P_{\text {incoh }} \approx \frac{\Gamma_{\mathrm{r}}}{2}$ and $P_{\text {loss }} \approx \frac{\Gamma_{\mathrm{n}}}{2}$. In this case, almost all the incoming

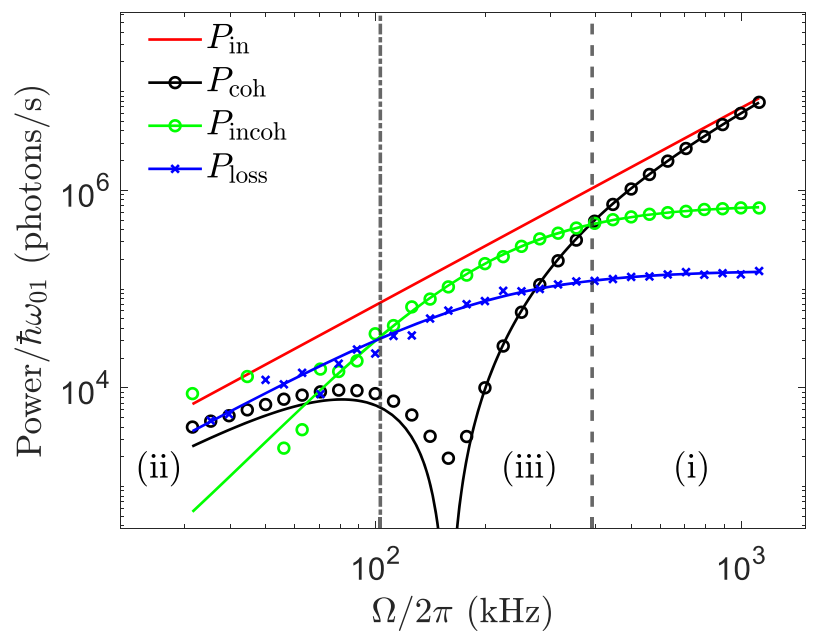

Fig. 5 Normalized powers as a function of Rabi frequency. The input power, $P_{\text {in }}$ (red), representing the input photon flux at the qubit, is measured when the qubit is tuned away by the external flux. When the qubit is on resonance with the input signal, we have the coherent power, $P_{\text {coh }}$ (black circles), consisting of photons reflected from either the qubit or the end of the transmission line. The qubit can also scatter photons incoherently, $P_{\text {incoh }}$ (green circles), due to the decoherence of the qubit. Moreover, the excited qubit has some probability to release a photon to the environment resulting in the power loss, $P_{\text {loss }}$ (blue crosses). The solid curves are fits to different types of the scattered powers. The dotted and dashdotted lines separate the qubit response into three interesting regions (see more details in the text). photons are reflected by the mirror.

(ii) In the low-power region $\Omega<\sqrt{\frac{\Gamma_{1} \Gamma_{2} \Gamma_{n}}{\Gamma_{\mathrm{r}}-\Gamma_{\mathrm{n}}}} \approx 2 \pi * 103 \mathrm{kHz}$ derived from $P_{\text {incoh }}=P_{\text {loss, }}$ to the left of the dash-dotted line, the scattering process is dominated by the interaction between the qubit and the incoming photons. The incoherent scattering is proportional to $\rho_{11}^{2}$ whereas the power loss depends linearly on the excitation probability. Therefore, the incoherent power can be less than the power loss when $\rho_{11}<\frac{\Gamma_{\mathrm{n}}}{2 \Gamma_{\mathrm{r}}} \approx 0.11$. Besides the incoherent photons, there is a small coherent scattering by the qubit. Compared to the loss, the coherent power is smaller if the non-radiative decay is large enough, namely if $\Gamma_{\mathrm{n}}>\frac{\Gamma_{1}\left(\Gamma_{\mathrm{r}}-\Gamma_{2}\right)^{2}}{2 \Gamma_{\mathrm{r}} \Gamma_{2}} \approx 2 \pi * 34 \mathrm{kHz}$.

(iii) In the intermediate-power region where $\sqrt{\frac{\Gamma_{1} \Gamma_{2} \Gamma_{n}}{\Gamma_{r}-\Gamma_{n}}}<\Omega<\left(1+\frac{1}{\sqrt{2}}\right) \Gamma_{r}$, both the mirror and the qubit make substantial contributions to the scattering process. The photons reflected by the mirror interfere destructively with those scattered by the qubit, resulting in a suppression of the coherent part of the output field. In particular, the dip around $\Omega / 2 \pi \approx 160 \mathrm{kHz}$ in the coherent power appears due to the fully destructive interference. In addition, the qubit excitation is not small anymore and the incoherent power is larger than the loss because $\Gamma_{r}>\Gamma_{n}$. We note that this region can be non-existent when either the non-radiative decay or the pure dephasing is sufficiently large.

We also fit the data in Fig. 5 to obtain all the decay rates. The result for the incoherent power indicates $\Gamma_{\mathrm{r}} / 2 \pi=229 \pm 2 \mathrm{kHz}$ with $\Gamma_{1} \Gamma_{2} / 4 \pi^{2}=39590 \pm 211 \mathrm{kHz}^{2}$ and $\Gamma_{1} \Gamma_{\phi} / 4 \pi^{2}=281 \pm 281 \mathrm{kHz}^{2}$. From fits to the power loss, we find $\Gamma_{n} / 2 \pi=49 \pm 1 \mathrm{kHz}$ and $\Gamma_{1} \Gamma_{2} / 4 \pi^{2}=$ $41260 \pm 4750 \mathrm{kHz}^{2}$. Therefore, with $\Gamma_{\mathrm{r}}$ and $\Gamma_{\mathrm{n}}$, we obtain $\Gamma_{1} / 2 \pi=$ $\left(\Gamma_{\mathrm{n}}+\Gamma_{\mathrm{r}}\right) / 2 \pi=278 \pm 2 \mathrm{kHz}$. Then, $\Gamma_{\phi} / 2 \pi \simeq 1 \mathrm{kHz}$. The coherent power yields $\Gamma_{\mathrm{r}} / 2 \pi=229 \pm 2 \mathrm{kHz}, \Gamma_{\mathrm{n}} / 2 \pi=48 \pm 8 \mathrm{kHz}$ and $\Gamma_{\phi} / 2 \pi=$ $1 \pm 1 \mathrm{kHz}$. Then, with $\Gamma_{1}$ and $\Gamma_{\phi}$, we have $\Gamma_{2} / 2 \pi=140 \pm 1 \mathrm{kHz}$.

From the discussion on region (i), at the highest Rabi frequency

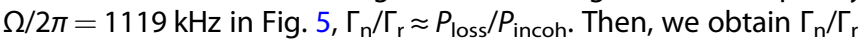
$=[0.1971,0.2385,0.2227,0.2297]$, by dividing the total measured data into four pieces. Combined with $\Gamma_{r}$ from the reflection coefficient in the section "Device characterization", we find $\Gamma_{\mathrm{n}} /$ $2 \pi \approx 45,54,51,52 \mathrm{kHz}$, respectively. The mean value is about $50 \mathrm{kHz}$ with $3 \mathrm{kHz}$ as the standard deviation. According to Eqs. (40) and (41) in Supplementary section $D$, as $\Gamma_{\phi}$ is small for our qubit, we have $\Gamma_{\mathrm{n}}=2 P_{\text {loss }}\left(1+\frac{\Gamma_{1} \Gamma_{2}}{\Omega^{2}}\right)$ and $\Gamma_{\mathrm{r}} \simeq 2 P_{\text {incoh }}\left(1+\frac{\Gamma_{1} \Gamma_{2}}{\Omega^{2}}\right)^{2}$. Due to $\frac{\Gamma_{1} \Gamma_{2}}{\Omega^{2}} \approx 3 \%$, we have $\Gamma_{\mathrm{n}} \approx 2.06 P_{\text {loss }}$ and $\Gamma_{r} \approx 2.12 P_{\text {incoh. }}$. Therefore, the estimated value for $\Gamma_{\mathrm{n}}$ has a systematic error of about $3 \%$. Since $\Gamma_{\phi}=\Gamma_{2}-\frac{\Gamma_{r}+\Gamma_{n}}{2}$ and $\Gamma_{1}=\Gamma_{r}+\Gamma_{n}$, the pure dephasing and total relaxation rates are $2 \pi^{*}(2 \pm 2) \mathrm{kHz}$ and $2 \pi^{*}(277 \pm 2) \mathrm{kHz}$, respectively. Additionally, because $P_{\text {loss }}=2 \pi^{*} 0.0243^{*} 10^{6}$ and $P_{\text {incoh }}=2 \pi^{*} 0.1056^{*} 10^{6}$, we have $\Gamma_{\mathrm{n}} / 2 \pi \approx 49 \mathrm{kHz}$ and $\Gamma_{\mathrm{r}} / 2 \pi \approx$ $224 \mathrm{kHz}$, respectively.

The results shown here agree well with the values from other sections in the paper, which implies that it is possible to take the $\Gamma_{r}$ value from the reflection coefficient measurement as a reference for the Mollow triplet in order to separate the non-radiative decay rate and the pure dephasing rate.

\section{Time-resolved dynamics}

All measurements described previously span time ranges from several hours to tens of hours. It is noteworthy that qubit decay rates extracted by different methods agree relatively well. However, the long duration means that any fluctuations of the decay rates are averaged out. Recently, several groups have characterized such fluctuations in circuit QED, using Rabi pulses, Ramsey interference measurements, and dispersive qubit readout $^{36-39,54}$. To probe the decoherence of the qubit with a temporal 

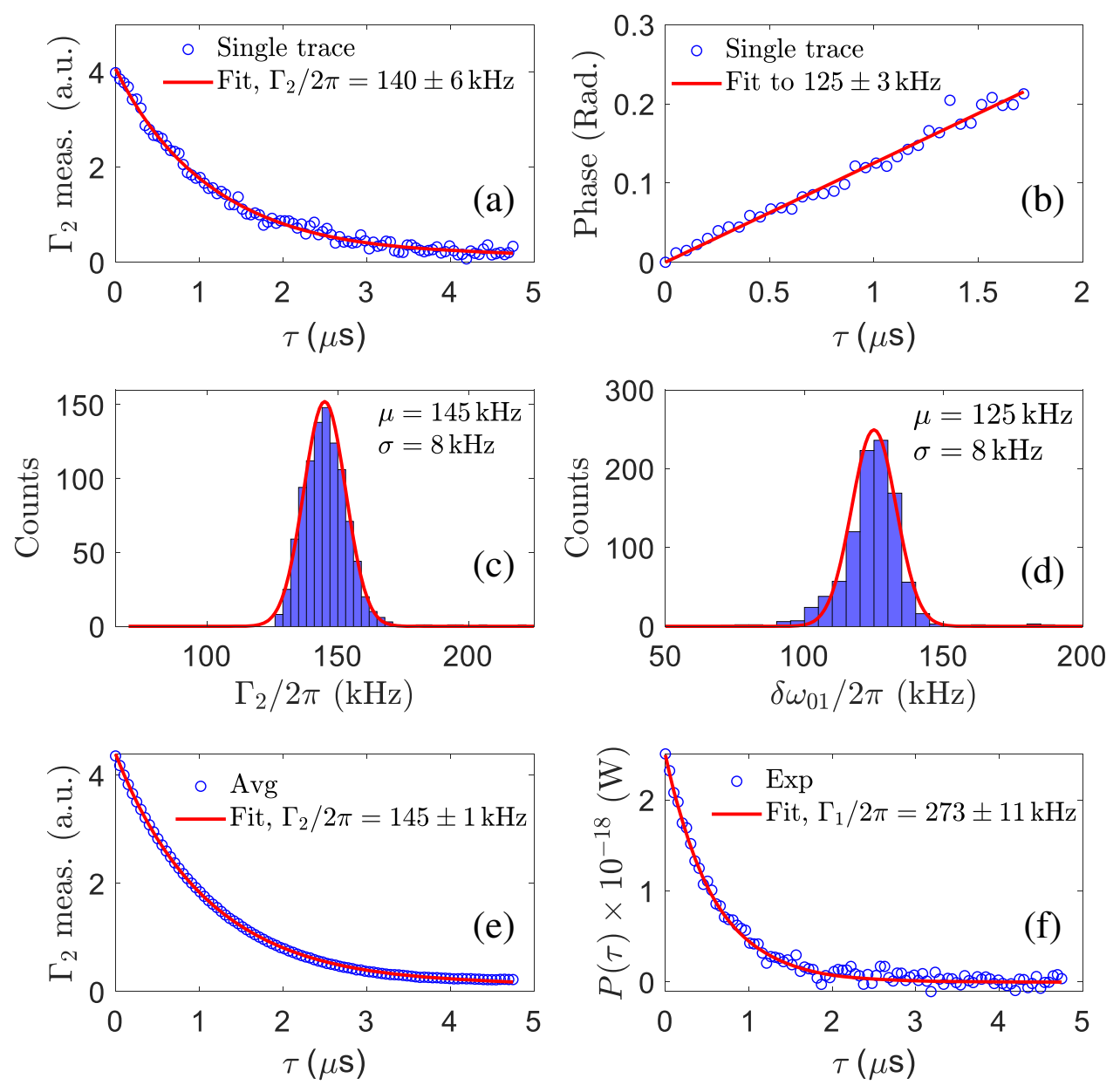

Fig. 6 Qubit dynamics to measure $\Gamma_{2}, \Gamma_{1}$ and $\delta \omega_{01} / 2 \pi$. The magnitude response of the measured signal is proportional to the magnitude of the emission operator $\left\langle\sigma_{-}\right\rangle$of the qubit while the phase response increases linearly with time as $\delta \omega_{01} \tau$. a A single trace of the magnitude response of $\Gamma_{2}$ measurements after a $\pi / 2$-pulse, showing the decoherence processes of the qubit within time $\tau$. The data is fitted to an exponential decay. $\mathbf{b}$ The corresponding phase response from (a), showing that the phase of the emitted photon from the qubit evolves with a slope corresponding to the detuning $\delta \omega_{01} / 2 \pi=125 \mathrm{kHz}$ where $\delta \omega_{01}=\omega_{01}-\omega_{\text {pulse. }}$. Histogram of $\Gamma_{2}$ from the magnitude response of the measurements from 975 traces, spanning $4.27 \times 10^{5} \mathrm{~s}$ (approximately $119 \mathrm{~h}$ ). $\mathbf{d}$ Histogram of $\Gamma_{2}$ from the corresponding phase response of the measurements taken in (c). Both (c) and (d) have been fitted (solid line) to a Gaussian distribution with parameters shown. e The decay of the qubit state by averaging all the measured traces in (c) to extract the decoherence rate. $\mathbf{f} A \pi$-pulse is applied to flip the qubit to the excited state with $1.92 \times 10^{9}$ averages, where $P(\tau)$ is the power emitted by the qubit at time $\tau$ after the pulse. By fitting the emitted power (blue circles) to an exponential decay, we extract $\Gamma_{1} / 2 \pi=273 \pm 11 \mathrm{kHz}$. Except for the histograms, the error bars are for $95 \%$ confidence.

resolution of 7 minutes, we prepare the qubit in a superposition of the ground and the first-excited state and monitor its spontaneous emission into the waveguide by recording both quadratures of the output field with a digitizer as a complex trace in the time domain, i.e., $V(t)=I(t)+i Q(t)$. We measure for $4.27 \times 10^{5} \mathrm{~s}$ (approximately 119 hours) with 975 repetitions, and each trace has $2.30 \times 10^{6}$ averages. The averaged trace, $\langle V(t)\rangle$, is proportional to the qubit emission. In order to generate a pulse at the qubit frequency, a signal generator at the frequency $250 \mathrm{MHz}$ higher than the qubit frequency is mixed with a pulse with a $250 \mathrm{MHz}$ carrier frequency. This pulse is from an arbitrary waveform generator (AWG) where the bandwidth of the AWG is 1.25 giga samples per second.

In detail, after the $50 \mathrm{~ns}$ long $\pi / 2$-pulse, the qubit superposition state evolves in time $\tau$ as $\frac{1}{\sqrt{2}}\left(|0\rangle+\mathrm{e}^{-\Gamma_{2} \tau-\mathrm{i} \delta \omega_{01} \tau}|1\rangle\right)$ with $\delta \omega_{01}=\omega_{01}$ $-\omega_{\text {pulse }}$. The emitted field carries information about the qubit operator $\left\langle\sigma_{-}\right\rangle=\mathrm{e}^{-\Gamma_{2} \tau} \mathrm{e}^{-\mathrm{i} \delta \omega_{01} \tau}$, where the amplitude response and the phase response show the decoherence and the qubit frequency shift with $\tau$, respectively.

Figure $6 a, b$ shows the magnitude and phase response of $a$ single trace where the decay of the magnitude is fitted to an exponential curve and the phase of the photons emitted from the qubit grows linearly with time due to the free evolution of the qubit, where the slope determines $\delta \omega_{01} / 2 \pi=125 \mathrm{kHz}$. Figures $6 c$, $\mathrm{d}$ are histograms of $\Gamma_{2}$ and $\delta \omega_{01}$ for all the repetitions. Both histograms can be fitted to a Gaussian with parameters shown in the figures. In comparison with the decoherence rates extracted from other measurements, we find that the standard deviation here is larger than the previously measured error bar. This shows that the dynamics of the qubit on a short time differs slightly from that over a long measurement time. By taking the average of all the traces in Fig. $6 \mathrm{~d}$, we fit to an exponential decay and get an averaged $\Gamma_{2} / 2 \pi=145 \pm 1 \mathrm{kHz}$.

To study $\Gamma_{1}$, we instead send a $\pi$-pulse to fully flip the qubit, and then measure the emission from the qubit. The corresponding output power, $P(\tau)=\left(\hbar \omega_{01} \Gamma_{\mathrm{r}} / 2\right)\left(1+\left\langle\sigma_{z}\right\rangle\right) e^{-\Gamma_{1} \tau 55}$ allows us to determine $\Gamma_{1}$. The trace is measured with $1.92 \times 10^{9}$ averages, shown in Fig. 6e. A fit to an exponential decay with $\Gamma_{1} / 2 \pi=273 \pm$ $11 \mathrm{kHz}$ agrees well with the data. Combining these numbers with $\Gamma_{r}$ from section "Device characterization", we can also calculate $\Gamma_{\mathrm{n}}$ and $\Gamma_{\phi}$ from these measurements. The resulting values can are summarized in Table 1. 
Table 1. Summary of the different rates extracted using different methods.

\begin{tabular}{lllllllr}
\hline Method & $\begin{array}{l}\Gamma_{\mathrm{r}} / 2 \pi \\
(\mathrm{kHz})\end{array}$ & $\begin{array}{l}\Gamma_{\mathrm{n}} / 2 \pi \\
(\mathrm{kHz})\end{array}$ & $\begin{array}{l}\Gamma_{\phi} / 2 \pi \\
(\mathrm{kHz})\end{array}$ & $\begin{array}{l}\Gamma_{1} / 2 \pi \\
(\mathrm{kHz})\end{array}$ & $\begin{array}{l}\Gamma_{2} / 2 \pi \\
(\mathrm{kHz})\end{array}$ & $\begin{array}{l}\text { BW } \\
(\mathrm{MHz})\end{array}$ & $\begin{array}{r}T \\
(\mathrm{~h})\end{array}$ \\
\hline Reflection & $227(1)$ & - & - & - & $141(1)$ & 4 & 3 \\
On-res.MT & - & $48(7)$ & $3(4)$ & $275(7)$ & $141(2)$ & 2 & 8 \\
Off-res.MT & - & $48(6)$ & $3(3)$ & $275(6)$ & $140(3)$ & 5 & 23 \\
Scattering & $229(2)$ & $49(1)$ & $3(1)$ & $278(2)$ & $142(1)$ & 5 & 63 \\
SinglePoint & - & $50(3)$ & $2(2)$ & $277(2)$ & - & 5 & 2 \\
Dynamics & - & $46(11)$ & $9(5)$ & $273(11)$ & $145(1)$ & 20 & 23 \\
\hline
\end{tabular}

The first method is a measurement of the reflection coefficient under a weak probe. On-res.MT and Off-res.MT represent the on/off-resonant Mollow triplet from atomic fluorescence, respectively. The energy loss is estimated by calibrating the input power and measuring both the coherent and incoherent power scattered by the atom. Finally, timeresolved measurements of the decay from both a superposition state and the first-excited state were used. BW and $T$ are the measurement bandwidth and the total measurement time, respectively, for each method.

\section{DISCUSSION}

We have shown several methods to determine different decay rates of a qubit placed in front of a mirror. In principle, these methods can also be used when the qubit couples to a transmission line without a mirror, except for the scattering method, where the corresponding measurement taken on both the input and output ports is required.

The measured rates are consistent between methods within the error bars of two standard deviations corresponding to $95 \%$ confidence. The results are summarized in Table 1 . The reflection measurement is the baseline to provide the value of $\Gamma_{r}$ to extract the non-radiative decay rate of the qubit for measurements, except for the scattering measurement. These different methods have advantages and disadvantages that we summarize below:

(i) The fastest way to obtain the non-radiative decay rate is to send a strong pump on resonance with the qubit so that the central peak and the sidebands of the Mollow triplet do not overlap. The drawback is that the pump power needed here is much stronger than for the other methods and that may change the rates slightly.

(ii) In the second method, we measure the off-resonant Mollow triplet by detuning the pump frequency slightly from the qubit frequency. The sidebands will be asymmetric around the central peak if the pure dephasing rate is non-negligible. In this case, only weak probe power is required. However, the corresponding measurement time is increased by almost a factor of three.

(iii) The most accurate way to measure the non-radiative decay rate is to measure the difference between the input and output power, labeled as Scattering in Table 1. Using this method, we can obtain not only the power loss but also the coherent and incoherent power scattered by the qubit. However, the measurement time is much longer. In addition, the attenuation between the sample and the input line, as well as the gain between the detector and the sample have to be calibrated at the beginning, in order to get the absolute power values from the qubit. To simplify the measurement, as we described in "Photon scattering by the qubit", we can see that when the pump saturates the qubit, we get $P_{\text {incoh }} \approx \frac{r_{r}}{2}$ and $P_{\text {loss }} \approx \frac{r_{n}}{2}$. Then, the ratio of the non-radiative decay rate to the radiative decay rate can be obtained from the ratio of the lost power to the incoherent power. Knowing the value of $\Gamma_{r}$ from the reflection measurement, we can obtain the non-radiative decay rate. Therefore, in principle, we do not need to sweep the pump power as was done in Fig. 5. This simple way is labeled as SinglePoint in Table 1.

(iv) Finally, pulses can be applied to excite the qubit. Afterward, the exponential decay of the emission and the emitted power trace from the qubit can be recorded to extract the total relaxation rate and the decoherence rate with a much larger measurement bandwidth. The distortion on the scattered photons due to the non-flat frequency response will affect the extracted values of the decay rates. This may be the reason why decay rates from this method are slightly different from those measured by other methods. However, the advantage of this method is that it allows us to study the short-time dynamics of the qubit.

The measurement time for these methods is from 2 to $63 \mathrm{~h}$. The coherent measurement is related to the first moment (amplitude) whereas other methods are related to the second moment (power). In order to estimate the system noise $N$, we measured the background PSD by turning the drive off (not shown) and comparing the result with the measurement of Fig. 2c. We found $N \approx 49$ photons. However, we expect that using a quantum-limited Josephson traveling-wave amplifier ${ }^{56}$ would reduce the system noise to about two photons. The corresponding SNR is improved by a factor of 5 . Therefore, this would result in a reduction of the measurement time by factors of 25 and 625 , respectively, for the coherent measurement and the other methods ${ }^{57}$.

From the measured result, our qubit is $T_{1}$-limited, i.e., the radiative decay dominates the interaction. However, the nonradiative decay rate is one order of magnitude larger than the pure dephasing rate. The corresponding spontaneous-emission factor is $\beta \approx 85 \%$, which is typically close to $100 \%$ when we engineer the radiative decay much larger than the non-radiative decay. Therefore, reducing the non-radiative decay rate will be the next step to improve the intrinsic coherence of our qubit. In addition, it is worthwhile to investigate why the non-radiative decay rate of our qubit is one order of magnitude larger than the qubit coupled to a resonator which was fabricated on the same wafer ${ }^{38}$ in the future.

Our methods allow us to analyze all the decay channels in detail. This will be useful to study and engineer decay channels of the qubit, which is the critical element in superconducting circuits. For example, engineering the decay channels can improve the quantum efficiency of generating single photons, which is set by $\Gamma_{r} / 2 \Gamma_{2}$. Also, the fidelity of detecting a single photon can be increased by extending the qubit coherence time. More importantly, compared to circuit QED where a resonator dispersively couples to a qubit, our study provides a straightforward way to investigate superconducting qubits, which are crucial elements in superconducting quantum computers.

\section{DATA AVAILABILITY}

The data that supports the findings of this study are available from the corresponding authors upon reasonable request.

\section{CODE AVAILABILITY}

The code that supports the findings of this study is available from the corresponding authors upon reasonable request.

Received: 9 January 2020; Accepted: 2 November 2020; Published online: 16 February 2021

\section{REFERENCES}

1. Steffen, M., DiVincenzo, D. P., Chow, J. M., Theis, T. N. \& Ketchen, M. B. Quantum computing: an IBM perspective. IBM J. Res. Dev. 55, 13 (2011). 
2. Arute, F. et al. Quantum supremacy using a programmable superconducting processor. Nature 574, 505 (2019).

3. Barends, R. et al. Superconducting quantum circuits at the surface code threshold for fault tolerance. Nature 508, 500 (2014).

4. Gu, X., Kockum, A. F., Miranowicz, A., Liu, Y.-x \& Nori, F. Microwave photonics with superconducting quantum circuits. Phys. Rep. 718, 1 (2017).

5. Roy, D., Wilson, C. M. \& Firstenberg, O. Colloquium: strongly interacting photons in one-dimensional continuum. Rev. Mod. Phys. 89, 021001 (2017).

6. Astafiev, O. et al. Resonance fluorescence of a single artificial atom. Science 327, 840 (2010).

7. Hoi, I.-C. et al. Giant cross-kerr effect for propagating microwaves induced by an artificial atom. Phys. Rev. Lett. 111, 053601 (2013).

8. Van Loo, A. F. et al. Photon-mediated interactions between distant artificial atoms. Science 342, 1494 (2013).

9. Mirhosseini, M. et al. Cavity quantum electrodynamics with atom-like mirrors. Nature 569, 692 (2019).

10. Kockum, A. F., Miranowicz, A., De Liberato, S., Savasta, S. \& Nori, F. Ultrastrong coupling between light and matter. Nat. Rev. Phys. 1, 19 (2019).

11. Forn-Díaz, P. et al. Ultrastrong coupling of a single artificial atom to an electromagnetic continuum in the nonperturbative regime. Nat. Phys. 13, 39 (2017).

12. Kuzmin, R., Mehta, N., Grabon, N., Mencia, R. \& Manucharyan, V. E. Superstrong coupling in circuit quantum electrodynamics. npj Quantum Inform. 5, 20 (2019).

13. Kuhn, A., Hennrich, M. \& Rempe, G. Deterministic single-photon source for distributed quantum networking. Phys. Rev. Lett. 89, 067901 (2002).

14. Motes, K. R. et al. Linear optical quantum metrology with single photons: exploiting spontaneously generated entanglement to beat the shot-noise limit. Phys. Rev. Lett. 114, 170802 (2015).

15. Zhou, Y., Peng, Z., Horiuchi, Y., Astafiev, O. \& Tsai, J. Tunable microwave singlephoton source based on transmon qubit with high efficiency. Phys. Rev. Appl. 13, 034007 (2020).

16. Peng, Z., de Graaf, S. E., Tsai, J. \& Astafiev, O. Tuneable on-demand single-photon source in the microwave range. Nat. Commun. 7, 12588 (2016).

17. Forn-Diaz, P., Warren, C., Chang, C., Vadiraj, A. \& Wilson, C. On-demand microwave generator of shaped single photons. Phys. Rev. Appl. 8, 054015 (2017).

18. Pechal, M. et al. Superconducting switch for fast on-chip routing of quantum microwave fields. Phys. Rev. Appl. 6, 024009 (2016).

19. Gasparinetti, S. et al. Correlations and entanglement of microwave photons emitted in a cascade decay. Phys. Rev. Lett. 119, 140504 (2017).

20. Fan, B. et al. Breakdown of the cross-Kerr scheme for photon counting. Phys. Rev. Lett. 110, 053601 (2013).

21. Sathyamoorthy, S. R. et al. Quantum nondemolition detection of a propagating microwave photon. Phys. Rev. Lett. 112, 093601 (2014).

22. Inomata, K. et al. Single microwave-photon detector using an artificial $\Lambda$-type three-level system. Nat. Commun. 7, 12303 (2016)

23. Kono, S., Koshino, K., Tabuchi, Y., Noguchi, A. \& Nakamura, Y. Quantum nondemolition detection of an itinerant microwave photon. Nat. Phys. 14, 546 (2018).

24. Royer, B., Grimsmo, A. L., Choquette-Poitevin, A. \& Blais, A. Itinerant microwave photon detector. Phys. Rev. Lett. 120, 203602 (2018).

25. Sathyamoorthy, S. R., Stace, T. M. \& Johansson, G. Detecting itinerant single microwave photons. Comptes Rendus Physique 17, 756-765 (2016).

26. Besse, J.-C. et al. Single-shot quantum nondemolition detection of individual itinerant microwave photons. Phys. Rev. X 8, 021003 (2018).

27. Zheng, H., Gauthier, D. J. \& Baranger, H. U. Waveguide QED: Many-body boundstate effects in coherent and Fock-state scattering from a two-level system. Phys. Rev. A 82, 063816 (2010).

28. Sánchez-Burillo, E., Zueco, D., Martín-Moreno, L. \& García-Ripoll, J. J. Dynamical signatures of bound states in waveguide QED. Phys. Rev. A 96, 023831 (2017).

29. Calajó, G., Fang, Y.-L. L., Baranger, H. U. \& Ciccarello, F. et al. Exciting a bound state in the continuum through multiphoton scattering plus delayed quantum feedback. Phys. Rev. Lett. 122, 073601 (2019).

30. Paulisch, V., Kimble, H. \& González-Tudela, A. Universal quantum computation in waveguide QED using decoherence free subspaces. New J. Phys. 18, 043041 (2016).

31. Zheng, H., Gauthier, D. J. \& Baranger, H. U. Waveguide-QED-based photonic quantum computation. Phys. Rev. Lett. 111, 090502 (2013).

32. Knill, E., Laflamme, R. \& Milburn, G. J. A scheme for efficient quantum computation with linear optics. Nature 409, 46 (2001).

33. Wen, P. Y. et al. Large collective Lamb shift of two distant superconducting artificial atoms. Phys. Rev. Lett. 123, 233602 (2019).

34. Wen, P. et al. Reflective amplification without population inversion from a strongly driven superconducting qubit. Phys. Rev. Lett. 120, 063603 (2018).

35. Hoi, I.-C. et al. Probing the quantum vacuum with an artificial atom in front of a mirror. Nat. Phys. 11, 1045 (2015).

36. Müller, C., Lisenfeld, J., Shnirman, A. \& Poletto, S. Interacting two-level defects as sources of fluctuating high-frequency noise in superconducting circuits. Phys. Rev. B 92, 035442 (2015).
37. Klimov, P. et al. Fluctuations of energy-relaxation times in superconducting qubits. Phys. Rev. Lett. 121, 090502 (2018).

38. Burnett, J. J. et al. Decoherence benchmarking of superconducting qubits. npj Quantum Inform. 5, 9 (2019).

39. Schlör, S. et al. Correlating decoherence in transmon qubits: low frequency noise by single fluctuators. Phys. Rev. Lett. 123, 190502 (2019).

40. Dunsworth, A. et al. Characterization and reduction of capacitive loss induced by sub-micron Josephson junction fabrication in superconducting qubits. Appl. Phys. Lett. 111, 022601 (2017)

41. Rao, V. M. \& Hughes, S. Single quantum-dot Purcell factor and $\beta$ factor in a photonic crystal waveguide. Phys. Rev. B 75, 205437 (2007).

42. Chu, D. Y. \& Ho, S.-T. Spontaneous emission from excitons in cylindrical dielectric waveguides and the spontaneous-emission factor of microcavity ring lasers. $J$. Opt. Soc. Am. B 10, 381 (1993).

43. Lecamp, G., Lalanne, P. \& Hugonin, J. Very large spontaneous-emission $\beta$ factors in photonic-crystal waveguides. Phys. Rev. Lett. 99, 023902 (2007).

44. Baba, T., Hamano, T., Koyama, F. \& Iga, K. Spontaneous emission factor of a microcavity dbr surface-emitting laser. IEEE J. Quantum Electron. 27, 1347 (1991).

45. Mollow, B. Power spectrum of light scattered by two-level systems. Phys. Rev. 188, 1969 (1969).

46. Toyli, D. et al. Resonance fluorescence from an artificial atom in squeezed vacuum. Phys. Rev. X 6, 031004 (2016).

47. Ulrich, S. et al. Dephasing of triplet-sideband optical emission of a resonantly driven inas/gaas quantum dot inside a microcavity. Phys. Rev. Lett. 106, 247402 (2011).

48. Roy, C. \& Hughes, S. Phonon-dressed Mollow triplet in the regime of cavity quantum electrodynamics: excitation-induced dephasing and nonperturbative cavity feeding effects. Phys. Rev. Lett. 106, 247403 (2011).

49. Koch, J. et al. Charge-insensitive qubit design derived from the Cooper pair box. Phys. Rev. A 76, 042319 (2007).

50. Autler, S. H. \& Townes, C. H. Stark effect in rapidly varying fields. Phys. Rev. 100, 703 (1955).

51. Probst, S., Song, F., Bushev, P., Ustinov, A. \& Weides, M. Efficient and robust analysis of complex scattering data under noise in microwave resonators. Rev. Sci. Instrum. 86, 024706 (2015).

52. Welch, P. The use of fast fourier transform for the estimation of power spectra: a method based on time averaging over short, modified periodograms. IEEE Trans. Audio Electroacoustics 15, 70 (1967).

53. Koshino, K. \& Nakamura, Y. Control of the radiative level shift and linewidth of a superconducting artificial atom through a variable boundary condition. New J. Phys. 14, 043005 (2012).

54. Goetz, J. et al. Second-order decoherence mechanisms of a transmon qubit probed with thermal microwave states. Quantum Sci. Technol. 2, 025002 (2017).

55. Abdumalikov Jr, A., Astafiev, O., Pashkin, Y. A., Nakamura, Y. \& Tsai, J. Dynamics of coherent and incoherent emission from an artificial atom in a 1D space. Phys. Rev. Lett. 107, 043604 (2011).

56. Macklin, C. et al. A near-quantum-limited Josephson traveling-wave parametric amplifier. Science 350, 307 (2015).

57. da Silva, M. P., Bozyigit, D., Wallraff, A. \& Blais, A. Schemes for the observation of photon correlation functions in circuit qed with linear detectors. Phys. Rev. A 82, 043804 (2010).

\section{ACKNOWLEDGEMENTS}

The authors acknowledge the use of Nano-fabrication Laboratory (NFL) at Chalmers. We wish to express our gratitude to David Niepce and Marco Scigliuzzo for insightful discussions. This work was supported by the Knut and Alice Wallenberg Foundation via the Wallenberg Center for Quantum Technology (WACQT) and by the Swedish ResearchCouncil and the EU-project OpenSuperQ.

\section{AUTHOR CONTRIBUTIONS}

Y.L. and P.D. planned the project. Y.L. performed the measurements with the input from A.B., J.B., S.G., and B.S. A.B. and Y.L. designed the sample, A.B. fabricated the device. A.F.R. worked on the recipe development and characterization. Y.L., E.W., A.F. K., S.G., and G.J. developed the theoretical expressions. All authors contributed to discussions and the interpretation of results. The manuscript was written by Y.L. with help from all authors. P.D. supervised this work.

\section{FUNDING}

Open Access funding provided by Chalmers University of Technology. 


\section{COMPETING INTERESTS}

The authors declare no competing interests.

\section{ADDITIONAL INFORMATION}

Supplementary information The online version contains supplementary material available at https://doi.org/10.1038/s41534-021-00367-5.

Correspondence and requests for materials should be addressed to Y.L. or P.D.

Reprints and permission information is available at http://www.nature.com/ reprints

Publisher's note Springer Nature remains neutral with regard to jurisdictional claims in published maps and institutional affiliations.
Open Access This article is licensed under a Creative Commons Attribution 4.0 International License, which permits use, sharing, adaptation, distribution and reproduction in any medium or format, as long as you give appropriate credit to the original author(s) and the source, provide a link to the Creative Commons license, and indicate if changes were made. The images or other third party material in this article are included in the article's Creative Commons license, unless indicated otherwise in a credit line to the material. If material is not included in the article's Creative Commons license and your intended use is not permitted by statutory regulation or exceeds the permitted use, you will need to obtain permission directly from the copyright holder. To view a copy of this license, visit http://creativecommons. org/licenses/by/4.0/.

(c) The Author(s) 2021 